reinen Salzen im wesentlichen nur die auf Zwischengitterplätzen sitzenden Silberionen beweglich sind. In Bleichlorid und Bleibromid dagegen beruht die Stromleitung auf der Beweglichkeit der Anionenleerstellen ${ }^{29,} \mathbf{3 0}$.

Da das Vorzeichen der Überführungswärme der Sauerstoffionen nach Tab. 1 auch bei den beiden Mischoxiden

$$
\left(\mathrm{ZrO}_{2}\right)_{0,88}(\mathrm{CaO})_{0,12} \text { und }\left(\mathrm{ZrO}_{2}\right)_{0,91}\left(\mathrm{Y}_{2} \mathrm{O}_{3}\right)_{0,09}
$$

mit großer Wahrscheinlichkeit negativ ist und da die Sauerstoffionen um einige Größenordnungen beweglicher sind als die Kationen und Elektronen ${ }^{8-11}$, folgt, daß die Sauerstoffionen über Sauerstoffionenleerstellen wandern. Dieser Befund steht im Einklang mit den Vorstellungen von $\mathrm{W}_{\text {AgNeR }}{ }^{6}$ über den Leitungsmechanismus in kubisch stabilisiertem Zirkonoxid und mit den aus röntgenographischen und pyknometrischen Dichtebestimmungen von Hund ${ }^{7}$ folgenden Vorstellungen über den Fehlordnungstyp derartiger Mischoxide.

29 W. SEIth, Z. Phys. 56, 802 [1929] ; 57, 869 [1929].

30 Z. Gyulai, Z. Phys. 67, 812 [1931].

\title{
Über die Kinetik der Wasserstoffpermeation durch Nickel
}

\author{
W. FISCHER \\ Physikalisches Laboratorium der AG. Brown, Boveri \& Cie., Baden, Schweiz \\ (Z. Naturforschg. 22 a, 1581-1586 [1967] ; eingegangen am 1. Juni 1967)
}

\begin{abstract}
The permeation rate of hydrogen through nickel foils of various thicknesses $(6,7 \mu \leqq d \leqq 1,83 \mathrm{~mm})$ has been measured in the temperature range $423^{\circ} \mathrm{K} \leqq T \leqq 1000^{\circ} \mathrm{K}$. The permeation rate $P\left[\mathrm{Mol} \mathrm{H}{ }_{2} \cdot \mathrm{cm}^{-1} \cdot \mathrm{s}^{-1}\right]$ under stationary conditions is inversely proportional to the thickness of the foil over the whole temperature range. The experimental results are fitted by the relation

$$
P=\left(7,22 \cdot 10^{-7} / d\right) \cdot \exp (-52400 / R T) .
$$

Diffusion contants $D\left[\mathrm{~cm}^{2} \cdot \mathrm{s}^{-1}\right]$ have been determined by means of the time lag method and have been calculated from steady state permeation rates and from hydrogen solubility data. The results are in accordance within the limits of error.

It is concluded that the rate determining step for the permeation is diffusion of hydrogen in nickel. Surface reactions are very fast.
\end{abstract}

Die Kinetik der Permeation eines Gases durch einen Festkörper wird im Allgemeinfall sowohl durch die Volumendiffusion im Festkörper als auch durch die an den Oberflächen ablaufenden Vorgänge (Adsorption und Desorption, Dissoziation in Atome oder Ionen) bestimmt. In vielen Fällen jedoch ist entweder der eine oder der andere Vorgang geschwindigkeitsbestimmend. Zum Beispiel verlaufen beim Durchtritt eines Gases durch eine Kunststoffmembran die Oberflächenreaktionen viel schneller als die Diffusion ${ }^{1-4}$, während beim Durchtritt von Wasserstoff durch eine Palladiummembran unter gewissen experimentellen Bedingungen die Oberflächenreaktionen langsam verlaufen im Vergleich zur Diffusion ${ }^{5-7}$.

1 R. M. Barrer, Trans. Faraday Soc. 35, 628 [1939].

2 R. M. Barrer u. G. Skirrow, J. Polymer Sci. 3, 549 [1948].

3 D. Jeschke u. H. A. Stuart, Z. Naturforschg. 16 a, 37 [1961].

4 A. S. Michaels, W. R. Vieth u. J. A. Barrie, J. Appl. Phys. 34, 1, 13 [1963].
Obgleich die Permeation von Wasserstoff durch Nickel von vielen Experimentatoren gemessen wurde, geht doch aus den bisher gewonnenen Meßdaten nicht eindeutig hervor, welcher Vorgang geschwindigkeitsbestimmend ist. Borelius und LindBLom $^{8}$ und Lombard ${ }^{9}$ finden, daß die Menge des durchtretenden Wasserstoffs der Dicke des Nickels umgekehrt proportional ist. Die Dicken werden jedoch nur um maximal $50 \%$ variiert. Außerdem zeigt sich, daß die aus der Wasserstofflöslichkeit im Nickel und aus Permeationsmessungen verschiedener Autoren berechneten Diffusionskoeffizienten von Wasserstoff in Nickel sehr nahe beieinander liegen (vgl. Abb. 4). Beide Befunde sprechen dafür, daß die Permeation im wesentlichen durch die Diffusion bestimmt wird.

5 R. M. Barrer, Trans. Faraday Soc. 36, 1235 [1940].

6 A. FARKAS, Trans. Faraday Soc. 32, 1667 [1936].

7 P. A. Silberg u. C. H. Bachman, J. Chem. Phys. 29, 777 [1958].

8 G. Borelius u. S. Lindblom, Ann. Phys. 82, 201 [1927].

9 V. M. Lombard, C. R. Acad. Sci. Paris 177, 116 [1923]. 
Smithells und Ransley ${ }^{10}$ konnten durch Oxydieren und Reduzieren der Oberfläche die Permeation um den Faktor 2 erhöhen. Umgekehrt kann die Durchlässigkeit von Nickel für Wasserstoff nach Experimenten von BAUKLOH und KAYSER durch längere Wärmebehandlung bis um den Faktor 6 verringert werden ${ }^{11}$. Diese Befunde sprechen dafür, daß die Oberflächenreaktionen für die Geschwindigkeit des Wasserstoffdurchtritts mitbestimmend sind.

Eine Klärung der Frage, in welchem Maße Diffusion und Oberflächenreaktionen an der Permeation von Wasserstoff durch Nickel beteiligt sind, wird durch Messung der Permeation in Abhängigkeit von der Dicke der Nickelschicht in einem größeren Bereich möglich. Außerdem kann der Diffusionskoeffizient von Wasserstoff in Nickel durch Verfolgen des nichtstationären Einstellvorganges bei der Permeation bestimmt werden. Über derartige Messungen wird berichtet.

\section{Meßanordnung}

Die zu untersuchende Nickelfolie ist so zwischen zwei Flansche eingeschweißt, daß zwei getrennte, gegen die Atmosphäre dichte Räume entstehen. In einem Raum wird ein bestimmter Wasserstoffdruck aufrechterhalten, während das durch die Folie in den anderen Raum diffundierende Gas von einem Pumpenaggregat abgepumpt und in einem bekannten Volumen gesammelt wird. Aus dem Druckanstieg in diesem Volumen kann die durch die Folie tretende Gasmenge berechnet werden.

Einzelheiten der Meßanordnung sind aus Abb. 1 ersichtlich. Der Wasserstoff strömt aus der Gasflasche über ein Magnetventil solange in das Druckregelvolumen, bis der untere Kontakt des Hg-Schaltmanometers mit dem oberen, angeschlossenen Kontakt über das Quecksilber verbunden wird. Durch die Spule des Magnetventils fließt von da an ein Strom, das Ventil schließt und eine weitere Gaszufuhr aus der Flasche wird unterbunden. Wenn der Druck soweit gesunken ist, daß der obere Kontakt am Schaltmanometer wieder öffnet, strömt erneut Gas ein. Die auf diese Weise erzielbare Druckregelgenauigkeit besträgt \pm 1 Torr. Der im Druckregelvolumen befindliche Wasserstoff wird einerseits durch Öffnen der Hähne $\mathrm{H}_{1}$ und $\mathrm{H}_{4}$ (oder $\mathrm{H}_{2}$ und $\mathrm{H}_{3}$ ) durch die wasserstoffdurchlässige Folie mittels der Diffusionspumpe, andererseits durch Öffnen des Hahnes $\mathrm{H}_{9}$ durch die Kapillare mittels der Vor-

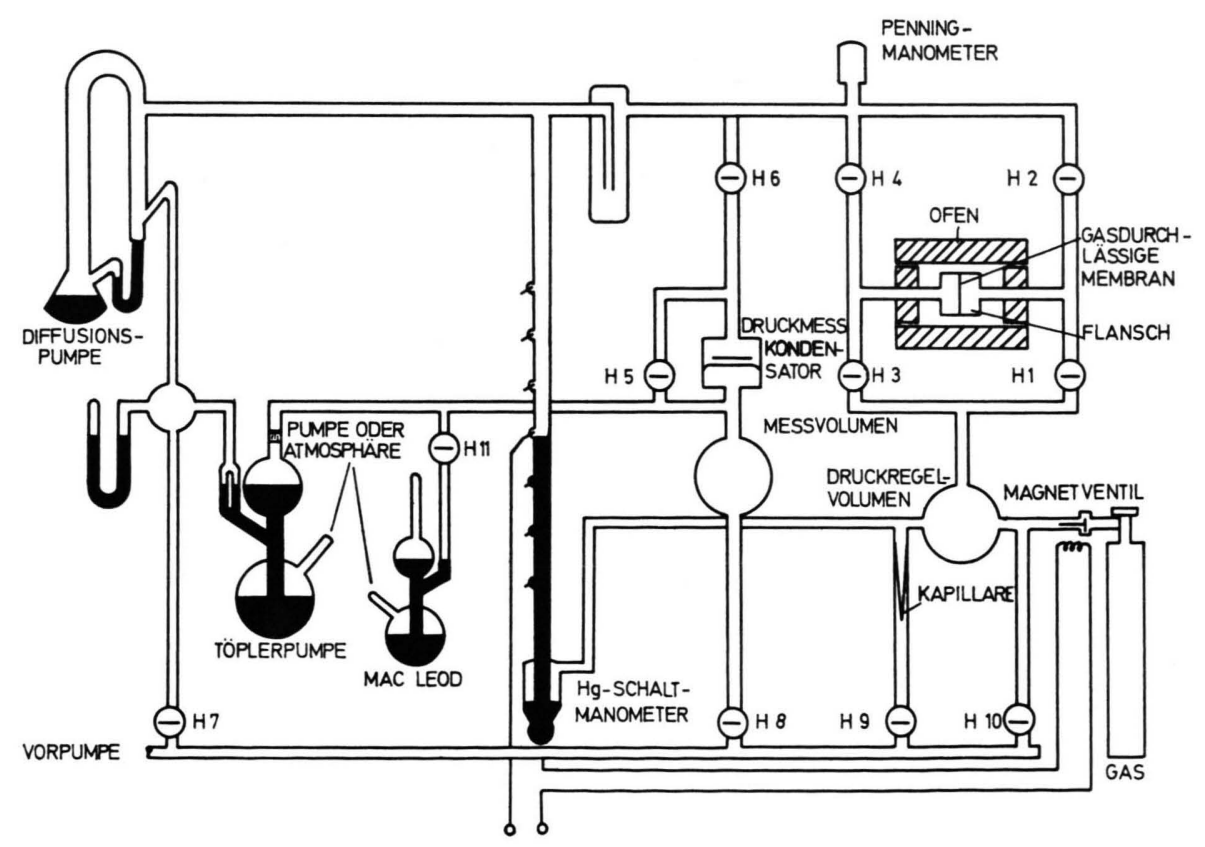

Abb. 1. Meßapparatur.

10 C. J. Smithells u. C. E. Ransley, Proc. Roy. Soc. London A 150, 172 [1935].
11 W. BaUkloh u. H. Kayser, Z. Metallk. 26, 157 [1934] ; 27, 281 [1935]. 
pumpe abgesaugt. Dadurch wird eine Anreicherung von Verunreinigungen im Wasserstoff auf der Druckseite der Folie vermieden.

Der durch die Folie tretende Wasserstoff wird von der Diffusionspumpe in das Vorvakuumvolumen und von der automatisch arbeitenden Töpler-Pumpe bei geschlossenem $\mathrm{Hahn} \mathrm{H}_{7}$ in das kalibrierte Meßvolumen gepumpt. Dieses wird am oberen Ende der Töpler-Pumpe durch eine Fritte G 4, die mit dem darüber stehenden Quecksilber ein Rückschlußventil bildet, abgeschlossen. Während des Meßvorganges sind die Hähne $\mathrm{H}_{5}$ und $\mathrm{H}_{8}$ geschlossen, der Hahn $\mathrm{H}_{6}$ ist geöffnet. Der Druck in der Hochvakuumleitung ist bei allen Messungen kleiner als $10^{-3}$ Torr, so $\mathrm{da} ß$ mit dem Druckkondensator (A t las - MembranKapazitäts-Torrmeter „MCT“, Meßbereich $10^{-2}$ bis 20 Torr) Absolutdrücke gemessen werden. Der Meßwert wird auf einen Schreiber gegeben, der dann direkt den zeitlichen Druckanstieg im Meßvolumen registriert.

Die Anlage arbeitet mit einer Verzögerung von etwa 30 Sekunden. Sie ist hauptsächlich durch die endliche Arbeitsfrequenz der TöPLER-Pumpe bedingt. Die Empfindlichkeit der Apparatur beträgt etwa $10^{-11} \mathrm{Mol}$ Gas pro sec.

Die Nickelfolien und -bleche wurden mit einer Elektronenstrahlschweißmaschine zwischen zwei Flansche geschweißt, wobei die 6,7 und $30 \mu$ dicken Folien durch ein Drahtnetz gestützt wurden. Die für die Permeation wirksamen Flächen wurden nach der Messung durch Auseinandersägen der Flansche bestimmt. Sie lagen zwischen 7,5 und $10,5 \mathrm{~cm}^{2}$.

Die Folien und Bleche enthielten mindestens 99,8\% Nickel. An Verunreinigungen waren Eisen $(0,04 \%)$, Mangan $(0,01 \%)$ und Silicium $(0,01 \%)$ sowie Magnesium, Kupfer, Calcium, Barium, Strontium und Kohlenstoff in Spuren vorhanden. Der Wasserstoff wurde zum größten Teil handelsüblichen Wasserstoffflaschen entnommen. Einige Messungen wurden mit Wasserstoff durchgeführt, der mit Hilfe eines Palladiumdiffusionsventils von Verunreinigungen befreit wurde.

\section{Meßergebnisse}

Mit der Apparatur wurde einerseits der im stationären Zustand durch die Nickelfolie fließende Wasserstoffstrom gemessen, andererseits wurde der Einstellvorgang verfolgt.
Für die Bestimmung der stationären Permeationsrate wurde auf der Druckseite der Folie ein Wasserstoffdruck von 760 Torr eingestellt. Die aus dem Druckanstieg und der Temperatur im Meßvolumen und aus der Fläche der Folie berechnete Permeation $P\left[\mathrm{Mol} \mathrm{H}_{2} \cdot \mathrm{cm}^{-2} \cdot \mathrm{s}^{-1}\right]$ ist in $\mathrm{Abb} .2$ für verschieden dicke Folien logarithmisch gegen die reziproke Temperatur aufgetragen. Die als Kreise eingezeichneten Meßpunkte wurden mit Wasserstoff aus handelsüblichen Wasserstoffflaschen, die als Kreuze eingezeichneten Meßpunkte mit gereinigtem Wasserstoff auf der Druckseite der Folie aufgenommen. Die Reinigung erfolgte mit einem Palladiumdiffusionsventil.

Vor und nach jeder Meßreihe wurde der Nulleffekt gemessen, das heißt der Gasstrom, der sich einstellt, wenn sich auf der Druckseite der Folie 760 Torr Helium befinden. Der Nulleffekt betrug maximal 5\% der zu messenden Wasserstoffpermeation, meist war er wesentlich kleiner.

Die Meßergebnisse werden durch die Relation

$$
P=\left(7,22 \cdot 10^{-7} / d\right) \exp (-52400 / R T)
$$

wiedergegeben, in welcher $P$ die Permeation in Mol $\mathrm{H}_{2} \cdot \mathrm{cm}^{-2} \cdot \mathrm{s}^{-1}, d$ die Dicke der Nickelfolie in $\mathrm{cm}, R$ $=8,314 \mathrm{~J} \cdot \mathrm{Mol}^{-1} \cdot \mathrm{Grad}^{-1}$ die Gaskonstante und $T$ die absolute Temperatur bedeuten.

Der nichtstationäre Einstellvorgang wurde an der $1,83 \mathrm{~mm}$ dicken Nickelscheibe verfolgt. Dazu wurde sie entgast, indem während einer Stunde auf beiden Seiten der auf $900{ }^{\circ} \mathrm{C}$ befindlichen Scheibe ein Vakuum von mindestens $10^{-4}$ Torr aufrechterhalten wurde. Vorversuche, bei denen das desorbierte Gas im Meßvolumen gesammelt wurde, hatten ergeben, $\mathrm{da} ß$ der Desorptionsvorgang nach einigen Minuten abgeschlossen ist.

Nach Einstellen der Meßtemperatur wurden beim Zeitpunkt 0 auf der Druckseite der Nickelscheibe 760 Torr Wasserstoff eingelassen. Der durch die Scheibe fließende Wasserstoffstrom und damit der Druckanstieg im Meßvolumen (siehe Abb.1) sind zunächst klein, da die Wasserstoffkonzentration in der Nickelscheibe am Anfang nur klein ist. Diese kleine Druckzunahme geht asymptotisch in die der stationären Permeation entsprechende lineare Druckzunahme im Meßvolumen über. Extrapoliert man in einer entsprechenden Darstellung den linearen Druckanstieg auf die Zeitachse, so erhält man auf dieser einen Achsenabschnitt $\tau$, welcher den Einstellvorgang charakterisiert. 


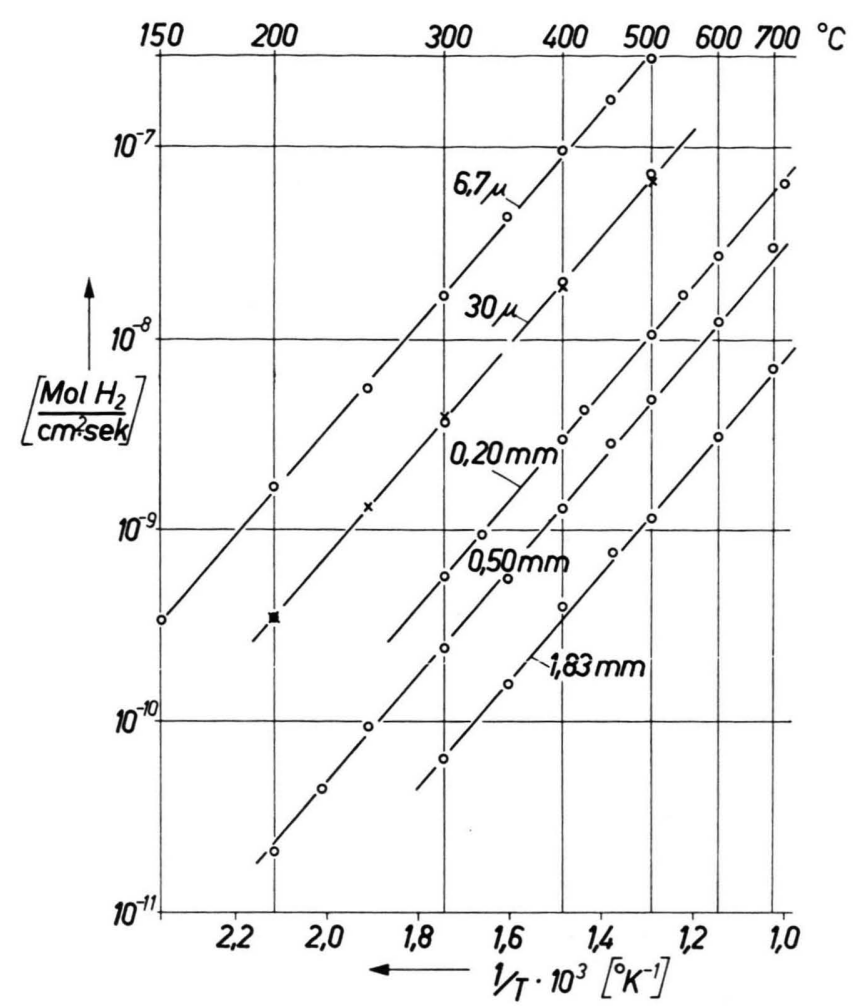

Abb. 2. Wasserstoffpermeation $\mathrm{P}\left[\mathrm{Mol} \mathrm{H}_{2} \cdot \mathrm{cm}^{-2} \cdot \mathrm{s}^{-1}\right]$ durch verschieden dicke Nickelfolien als Funktion der reziproken Temperatur. Als Parameter ist die Dicke der Folie angegeben.

Im Tab. 1 sind auf diese Weise bei verschiedenen Temperaturen ermittelte Zeiten $\tau$ zusammengestellt.

\begin{tabular}{lrrrrrrrr}
\hline$T\left[{ }^{\circ} \mathrm{C}\right]$ & 350 & 370 & 400 & 450 & 500 & 550 & 600 & 700 \\
$\tau[\mathrm{s}]$ & 2300 & 1860 & 1270 & 800 & 490 & 300 & 270 & 140
\end{tabular}

Tab. 1. Verzögerungszeiten $\tau$ beim Durchtritt von Wasserstoff durch eine $0,183 \mathrm{~cm}$ dicke Nickelscheibe.

Die Meßgenauigkeit betrug bei kleinen Einstellzeiten, also bei hohen Temperaturen $(550,600$, $700{ }^{\circ} \mathrm{C}$ ) wegen der endlichen Arbeitsfrequenz der Töpler-Pumpe nur 15 bis 30\%. Bei kleineren Temperaturen konnte $\tau$ mit einer Genauigkeit von etwa $10 \%$ bestimmt werden.

\section{Diskussion}

Für die Messung des stationären Wasserstoffstromes wurde auf der einen Seite der Nickelfolie ein

12 H. Deming u. B. Hendricks, J. Am. Chem. Soc. 45, 2857 [1923],

13 G. Euringer, Z. Phys. 96, 37 [1935].

14 C. B. Post u. W. R. Ham, J. Chem. Phys. 6, 598 [1938].

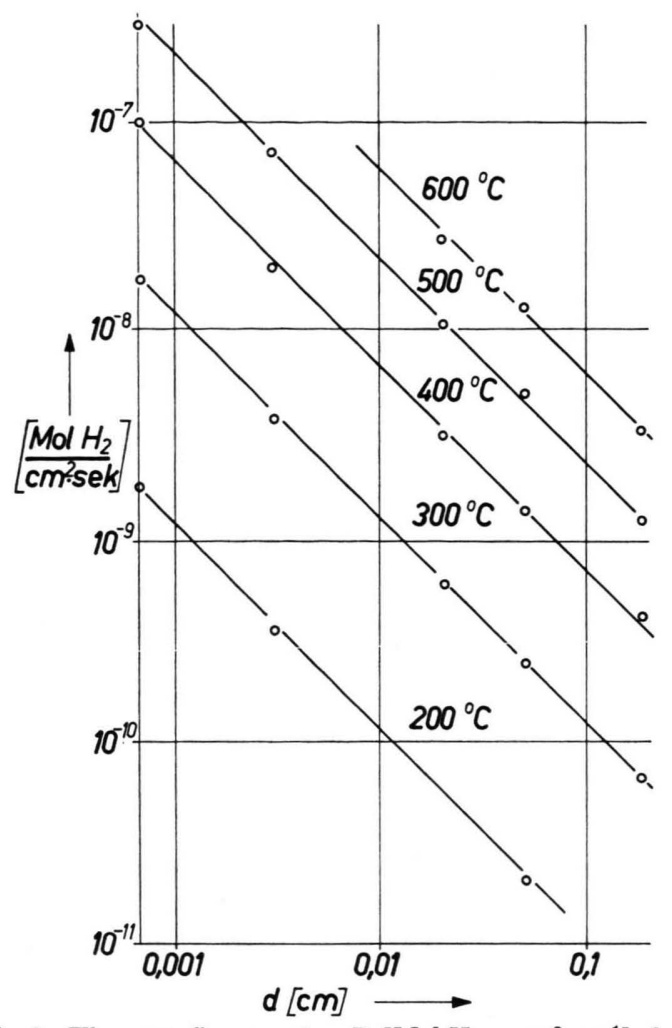

Abb. 3. Wasserstoffpermeation $P\left[\mathrm{Mol} \mathrm{H} \cdot \mathrm{cm}^{-2} \cdot \mathrm{s}^{-1}\right]$ durch Nickel als Funktion der Dicke $d[\mathrm{~cm}]$. Parameter: Temperatur.

Wasserstoffdruck von 760 Torr und auf der anderen Seite ein vernachlässigbar kleiner Druck aufrechterhalten. Für diesen Fall gilt, solange die Phasengrenzreaktionen an der Oberfläche des Nickels schnell ablaufen, das 1. Fick sche Gesetz in der einfachen Form

$$
P=D \cdot c / d \text {. }
$$

In Gl. (2) bedeutet $D$ den Diffusionskoeffizienten des Wasserstoffs im Nickel, $c$ die Wasserstofflöslichkeit im Nickel und $d$ die Dicke der Nickelfolie.

Nach Gl. (2) sollte man eine Gerade mit der Steigung -1 erhalten, wenn man $\log P$ gegen $\log d$ aufträgt. In Abb. 3 sind ein Teil der in Abb. 2 dargestellten Meßergebnisse entsprechend aufgetragen. Aus Abb. 3 ergibt sich, daß der erwähnte Zusammenhang für alle Temperaturen, bei denen genügend Meßpunkte vorhanden sind, gültig ist. Aus der Literatur ${ }^{8-18}$ und aus der Darstellung der Abb. 2

15 M. L. Hill u. E. W. Johnson, Acta Met. 3, 265 [1955].

16 A. G. Edwards, Brit. J. Appl. Phys. 8, 406 [1957].

17 H. Grimes, Acta Met. 7, 782 [1959].

18 W. Eichenauer, Mém. Sci. Rev. Met. 57, 943 [1960]. 
folgt also einerseits, daß die Temperaturabhängigkeit der Permeation durch die Gleichung

$$
P=K \exp \left(-E_{P} / R T\right)
$$

beschrieben wird, und aus der Darstellung der Abb. 3 folgt andererseits, daß die Permeation der Dicke der Nickelfolie umgekehrt proportional ist. Die Ergebnisse der Permeationsmessungen lassen sich deshalb durch eine einzige Gleichung, nämlich durch die Gl. (1) ausdrücken.

Die umgekehrte Proportionalität der Permeation zur Dicke der Nickelfolie bedeutet, daß die Phasengrenzreaktionen, also beispielsweise die der Volumendiffusion vorausgehende Adsorption des Wasserstoffs auf der Druckseite und die der Volumendiffu. sion nachfolgende Desorption des Wasserstoffs auf der Vakuumseite schnell verlaufen im Vergleich zur Diffusion. Nach Dünwald und W WGNER $^{19}$ kann man durch Verringerung der Dicke einer Folie im Prinzip immer erreichen, daß die Phasengrenzreaktionen für die Permeation geschwindigkeitsbestimmend werden. Nach dem Fick'schen Gesetz (2) kann man nämlich den Diffusionsstrom beliebig groß machen, wenn man nur die Folie genügend dünn wählt. Die Geschwindigkeit der Phasengrenzreaktionen hängt dagegen nicht von der Dicke der Folie ab. Danach folgt aus den Experimenten genauer, daß die Phasengrenzreaktionen selbst bei der dünnsten Nikkelfolie von $6,7 \mu$ noch so schnell ablaufen, daß ein Einfluß auf die Permeation nicht festgestellt werden kann.

Wegen der Gültigkeit des Zusammenhangs (2) kann aus der Permeation $P$ bei Kenntnis der Wasserstofflöslichkeit der Diffusionskoeffizient $D$ des Wasserstoffs im Nickel berechnet werden. Die Wasserstofflöslichkeit im Nickel ist aus Messungen von Sieverts und Hagenacker ${ }^{20}$ und von Armbruster ${ }^{21}$ bekannt. Die Meßergebnisse dieser Autoren genügen der Gleichung

$$
c=1,34 \cdot 10^{-4} \exp (-12400 / R T),
$$

aus welcher man die Konzentration $c$ in $\mathrm{Mol} \cdot \mathrm{cm}^{-3}$ erhält, wenn man die Gaskonstante $R$ in $\mathrm{J} \cdot \mathrm{Mol}^{-1}$. $\operatorname{grad}^{-1}$ einsetzt. Aus den Gln. (1) bis (3) ergibt sich für den Diffusionskoeffizienten

$$
D=5,39 \cdot 10^{-3} \exp (-40000 / R T) .
$$

19 H. Dünwald u. C. W [1934].

20 A. Sieverts u. J. Hagenacker, Ber. D. Chem. Ges. 42, 338 [1909].
Dieser aus den stationären Messungen folgende Ausdruck für den Diffusionskoeffizienten ist in Abb. 4 als ausgezogene Gerade zusammen mit der aus Meßdaten anderer Autoren folgenden Temperaturabhängigkeit des Diffusionskoeffizienten (gestrichelte Geraden) graphisch dargestellt.

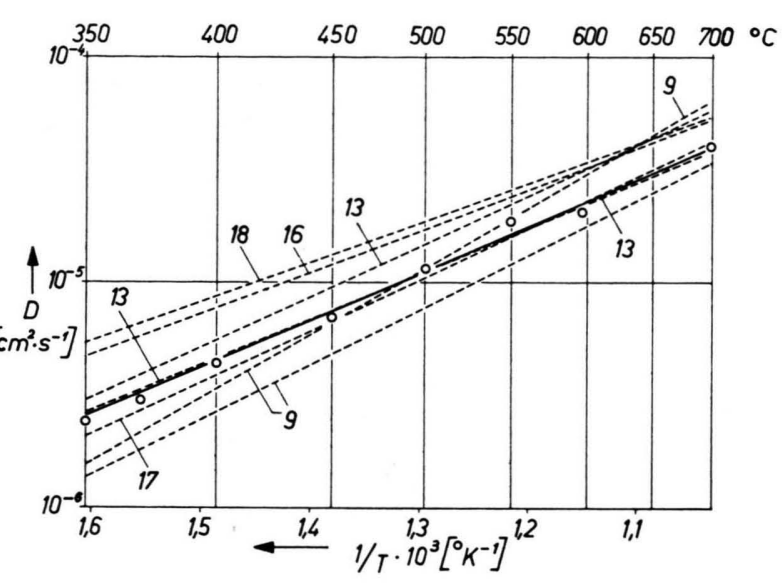

Abb. 4. Diffusionskoeffizient $D\left[\mathrm{~cm}^{2} \cdot \mathrm{s}^{-1}\right]$ von Wasserstoff in Nickel. Die ausgezogene Gerade wurde aus stationären Permeationsmessungen und aus Literaturwerten der Wasserstofflöslichkeit im Nickel berechnet. Die Kreise entsprechen den nach Gl. (5) aus instationären Messungen berechneten Werten für $D$. Die gestrichelten Geraden repräsentieren die nach verschiedenen Methoden gewonnenen Meßergebnisse mehrerer Autoren, wobei die Zahlen auf die entsprechenden Literaturzitate hinweisen.

Der Diffusionskoeffizient kann nicht nur aus den stationären, sondern auch aus den instationären Messungen ermittelt werden. Nach BarRer ${ }^{1,22}$ hängt der beim Einstellvorgang durch Extrapolation des stationären Druckanstiegs auf die Zeitachse gefundene Achsenabschnitt $\tau$ mit dem Diffusionskoeffizienten durch die Beziehung

$$
D=d^{2} / 6 \tau
$$

zusammen. Diese Formel gilt streng, solange die Kinetik der Permeation allein durch die Volumendiffusion bestimmt wird. Sie liefert aber auch dann noch eine gute Näherung, wenn die Phasengrenzreaktionen schon eine merkliche Rolle spielen ${ }^{5}$. Die nach Gl. (5) mit Hilfe der Meßergebnisse der Tab. 1 berechneten Diffusionskoeffizienten sind als Kreise

21 N. A. Armbruster, J. Am. Chem. Soc. 65, 1043 [1943].

22 R. M. Barrer, Phil. Mag. 28, 148 [1939]. 
in die graphische Darstellung der Abb. 4 mit aufgenommen. Die Übereinstimmung der nach dieser Methode ermittelten Diffusionskoeffizienten mit den aus den stationären Messungen und den Wasserstofflöslichkeiten berechneten Diffusionskoeffizienten ist ein weiterer Hinweis dafür, daß die Kinetik der Permea- tion allein durch die Volumendiffusion bestimmt wird.

Im gleichen Sinne läßt sich schließlich der Befund deuten, daß die im technischen Wasserstoff enthaltenen Verunreinigungen keinen Einfluß auf die Permeation haben.

\title{
Die solaren Bursts vom Typ III
}

\author{
R. WAGNER \\ Institut für Theoretische Physik und Sternwarte der Universität Kiel \\ (Z. Naturforschg. 22 a, 1586-1592 [1967] ; eingegangen am 14. März 1967)
}

\begin{abstract}
In a previous paper formulae for the radio emission originating from the scattering of a plane plasma electron wave by thermal fluctuations were derived. These formulae are applied to the solar type III bursts by starting from a cloud of electrons which trausverses the solar corona thereby exciting plasma waves. The electric field of the excited waves is estimated by an expression of the quasilinear theory. The band width of these waves is about $10 \%$ of the local plasma frequency. The scattering mechanism considered here can account only for the faint and moderate type III bursts without harmonic structure. The possibility to determine the thickness of the cloud in its variation with time is discussed.
\end{abstract}

In der vorangegangenen Arbeit *, die im folgenden mit I bezeichnet wird, wurde die Entstehung von Radiostrahlung in einem Plasma durch Streuung einer ebenen Plasmawelle an den thermischen Dichtefluktuationen des Plasmas diskutiert. Dazu wurden die Fluktuationen durch thermische Plasmaelektronen- und -ionenwellen dargestellt. Im Falle der Streuung an den thermischen Ionenwellen ergab sich eine Radioemission bei der Elektronenplasmafrequenz $\omega_{\mathrm{p}}$, während bei der Streuung an den thermischen Elektronenwellen Radiostrahlung bei $2 \omega_{\mathrm{p}}$ entstand. Die in I hergeleiteten Intensitätenformeln (92) und (63) werden im folgenden auf die Bursts vom Typ III angewandt. Dabei wird von der Tatsache ausgegangen, daß der Typ III durch Teilchenwolken hervorgerufen wird ${ }^{1,2}$. Diese Teilchenwolken müssen aus Elektronen bestehen. Die großen Stoßzeiten für Protonen in der Korona könnten die untere Grenze der radialen Geschwindigkeiten der Wolke $^{3}$ von $0,2 c \quad(c=$ Lichtgeschwindigkeit $)$ nicht erklären ${ }^{4,5}$.

Die Entstehung der Typ III-Radiostrahlung hat man sich nach WILD, SMerd und WeIss ${ }^{1}$ so vorzustellen: Die Elektronenwolke erregt über die Zwei-

* R. WAGNER, Z. Naturforschg. 22 a, 1372 [1967].

1 J. P. Wild, F. S. Smerd u. A. A. Weiss, Ann. Rev. Astron. Astrophys. 1, 291 [1963].

2 Y. Uchida, Publ. Astron. Soc. Japan 12, 376 [1960].

3 J. P. Wild, K. U. Sheridan u. A. A. Neylan, Australian J. Phys. 12, 369 [1959]. strominstabilität in der Korona Plasmawellen, die zum Teil in Radiowellen umgesetzt werden. Zur Berechnung der abgestrahlten Intensität aus (92) bzw. (93) und (63) benötigt man die elektrische Feldstärke dieser Plasmawellen.

\section{Elektrische Feldstärke der Plasmawellen}

Für ein stoßfreies Elektronenplasma mit verschmiertem Ionenhintergrund läßt sich nach KNORR ${ }^{6}$ die elektrische Feldstärke $E_{\mathrm{k}}$ einer Plasmawelle der Wellenzahl $k$ mit Hilfe der quasilinearen Theorie abschätzen. Setzt man die entsprechenden Größen des vorliegenden Problems ein, so erhält man unter der Voraussetzung, daß das System Korona - Wolke hinreichend gut durch eine Temperatur, die Koronatemperatur $T_{\mathrm{c}}$, beschrieben wird:

$$
\frac{E_{k}^{2}}{8 \pi}=\frac{n_{\mathrm{c}}}{2}(1+\mu)^{1,5} \varkappa T_{\mathrm{c}} \alpha \frac{\gamma^{3}}{k \lambda_{\mathrm{Dc}}}
$$

mit

$$
\begin{aligned}
\gamma=\frac{\pi}{2 k \lambda_{\mathrm{Dc}}}(1+\mu)^{-1 / 2}\left(\frac{\omega_{\mathrm{pc}}}{\omega}\right)^{2} \\
\cdot\left[v v_{\mathrm{Tc}}\left\{\frac{\partial f_{\mathrm{c}}}{\partial v}+\mu \frac{\partial f_{\mathrm{w}}}{\partial v}\right\}\right]_{v=\omega^{\prime} k} .
\end{aligned}
$$

4 C. DE JAGER, Space Research, Vol. I, ed. H. Kallmann Bijl, North Holland Publishing Co., Amsterdam 1960.

5 M. R. Kundu, Solar Radio Astronomy, Interscience Publishers, New York 1965.

6 G. KNORR, Z. Naturforschg. 18 a, 1304 [1963]. 